\title{
OPEN Global phylogeography of a pantropical mangrove genus Rhizophora
}

\author{
Koji Takayama ${ }^{1 \bowtie}$, Yoichi Tateishi² \& Tadashi Kajita $\mathbb{1}^{3,4 \bowtie}$ \\ Rhizophora is a key genus for revealing the formation process of the pantropical distribution of \\ mangroves. In this study, in order to fully understand the historical scenario of Rhizophora that \\ achieved pantropical distribution, we conducted phylogeographic analyses based on nucleotide \\ sequences of chloroplast and nuclear DNA as well as microsatellites for samples collected worldwide. \\ Phylogenetic trees suggested the monophyly of each AEP and IWP lineages respectively except for \\ $R$. samoensis and $R$. $\times$ selala. The divergence time between the two lineages was $\mathbf{1 0 . 6}$ million years \\ ago on a dated phylogeny, and biogeographic stochastic mapping analyses supported these lineages \\ separated following a vicariant event. These data suggested that the closure of the Tethys Seaway and \\ the reduction in mangrove distribution followed by Mid-Miocene cooling were key factors that caused \\ the linage diversification. Phylogeographic analyses also suggested the formation of the distinctive \\ genetic structure at the AEP region across the American continents around Pliocene. Furthermore, \\ long-distance trans-pacific dispersal occurred from the Pacific coast of American continents to the \\ South Pacific and formed F1 hybrid, resulting in gene exchange between the IWP and AEP lineages \\ after 11 million years of isolation. Considering the phylogeny and phylogeography with divergence \\ time, a comprehensive picture of the historical scenario behind the pantropical distribution of \\ Rhizophora is updated.
}

Mangrove forests are the most critical component of coastal and estuarine ecosystems covering over $150,000 \mathrm{~km}^{2}$ in 123 countries across the tropics and the subtropics ${ }^{1}$. They provide tremendous ecological services for human beings, however, deforestation of mangrove forests are ongoing worldwide by anthropogenic as well as climate changes $^{2,3}$. Conservation of mangrove forests is becoming a global concern, and local, regional and nation-wide conservation activities are prominent in the world, however, our understandings on the global mangrove are limited because studying mangrove across nations and continents over pantropical distribution was difficult ${ }^{4}$.

The present pantropical distribution of mangrove plants could be due to the long-distance dispersal of their diaspores (seed, fruit, or propagule) by ocean currents ${ }^{5}$. Late Cretaceous fossil evidence of mangroves ${ }^{6,7}$ suggested the presence of mangrove over the world at that time. However, the evolutionary process and regional diversification of mangrove species to form the present pantropical distribution of mangrove forests are poorly understood. Uncovering the formation process of the pantropical distribution of mangroves will help deeper understandings of the global mangrove and provide us new insight into future changes of the mangrove ecosystem under global climate change.

Previous studies inferred a historical process of the pantropical distribution formation by considering the interactions between sea-dispersal and geological history based on the distribution patterns and fossil records of mangroves ${ }^{5}$. Duke et al. ${ }^{8}$, in particular, suggested constructing the phylogenetic history of a pantropical genus Rhizophora L. (Rhizophoraceae), which is a key taxon in revealing the global and disjunctive distribution patterns of mangroves. The genus Rhizophora dominates most of the mangrove forests in both Indo-West Pacific (IWP) and Atlantic-East Pacific (AEP) regions ${ }^{9,10}$, and the fossil records of the genus and family are relatively abundant owing to the distinct shape of the propagules. By studying the fossil records of the genus in detail, a scenario in which two lineages of Rhizophora diversified for IWP and AEP lineages owing to the closure of the Tethys Seaway in the Oligocene was proposed ${ }^{5}$. This scenario was the most comprehensive one at that time; however, it was merely a deduction based on fossil records and geological history.

\footnotetext{
${ }^{1}$ Department of Botany, Graduate School of Science, Kyoto University, Kitashirakawa Oiwake-cho, Sakyo-ku, Kyoto 606-8502, Japan. 'Faculty of Education, University of the Ryukyus, Senbaru 1, Nakagami-gun, Okinawa 903-0129, Japan. ${ }^{3}$ Iriomote Station, Tropical Biosphere Research Center, University of the Ryukyus, 870 Uehara, Taketomi-cho, Yaeyama-gun, Okinawa 907-1541, Japan. ${ }^{4}$ United Graduate School of Agricultural Science, Kagoshima University, Kagoshima, Japan. ${ }^{\circledR}$ email: takayama@sys.bot.kyoto-u.ac.jp; kajita@mail.ryudai.jp
} 
Many mangrove researchers tried to understand the most intriguing question, that is, the historical process of formation of the world mangroves, focusing on the genus Rhizophora by using molecular markers ${ }^{11-16}$. Divergence times between the species in IWP and AEP regions have been estimated by using various molecular markers and sampling coverages. Lo et al. ${ }^{14}$ suggested that the divergence time between IWP and AEP lineages of Rhizophora was approximately $48( \pm 3)$ Ma (million years ago) using cpDNA, ITS, and ISSR markers based on samples collected from a relatively wide range of distribution, however, they used limited numbers of AEP samples and no samples from West Africa. Chen et al. ${ }^{15}$ estimated the divergence time to be approximately 12.7 Ma (95\% HPD (Highest Posterior Density), 10.4 Ma-15.5 Ma) using five nuclear genes based on less number of samples and used only two AEP samples from West Africa. Although it was not stated in the paper at all, a supplementary data of Xu et al. ${ }^{16}$ implied that the divergence time could be $10.8 \mathrm{Ma}$ (95\% credible interval $9.2 \mathrm{Ma}$ - $12.8 \mathrm{Ma}$ ) using 590 single-copy genes extracted from genomic data based on only one representative sample from the AEP region. Even though Chen et al. ${ }^{15}$, as well as Xu et al. ${ }^{16}$ provided more reasonable divergence time of the two potential lineages of Rhizophora based on longer nucleotide sequences than any other previous studies, their study was based on only one or two representative samples from AEP Rhizophora species without showing the most critical basis for the global distribution, that is, the monophyly of AEP and IWP lineages respectively.

To understand the comprehensive picture of the phylogeographic process of Rhizophora, phylogenetic studies including all species as well as samples covering distinct lineages and wide distribution range are essential. Multiple samples from the wide distribution range of the AEP region are particularly important because the monophyly of the AEP lineages has not been clarified by nuclear DNA data, even though the presence of considerably distinct lineages across the American continents and West Africa was reported based on cpDNA and microsatellite data ${ }^{13}$. Numbers of distinct lineages were differentiated on both Pacific and Atlantic sides of the American continents, but none of the previous studies on the global phylogeny of Rhizophora included those distinct lineages in their phylogenetic analyses.

The sibling relationship between Rhizophora samoensis in South Pacific Islands in the IWP region and Rhizophora mangle in the AEP region has long been an enigmatic question discussed based on their morphological similarity ${ }^{10}$, and have recently been answered by molecular analyses using cpDNA sequences and microsatellite ${ }^{13}$. Rhizophora samoensis has an identical haplotype with the one of R. mangle in the Pacific coast of the American continents, which suggests at least a single long-distance migration occurred across the East Pacific. This unusual trans-pacific long-distance dispersal might have provided the opportunity for the two genetically distinct lineages, IWP and AEP lineages, to meet in the South Pacific Islands. Previous studies reported the presence of a putative hybrid species, $R$. $\times$ selala, between the two distinct lineages ${ }^{12,14,17-19}$, however, the phylogeographic process of the hybrid formation and hybridization patterns across the South Pacific Islands are still in question.

In this study, to obtain the most comprehensive scenario of historical processes that shaped the present pantropical distribution of mangrove forests, we performed the following analyses based on global sampling, including all species of Rhizophora as well as regional representative populations over the world. We performed 1) phylogenetic analyses using both cpDNA and nuclear DNA markers to obtain robust phylogeny to test the monophyly of the AEP lineage of Rhizophora in particular, 2) phylogeographic analyses to understand the historical process over the broad distribution range using the same molecular markers, and 3) estimation of divergence time by cpDNA phylogeny to give a robust time scale of mangrove diversification followed by ancestral range estimation to know the formation history of the distribution range. In addition, to understand the patterns of hybrid formation between AEP and IWP lineages of Rhizophora at the South Pacific. We also performed 4) population genetic analyses by comparing cpDNA and nuclear DNA sequences plus microsatellite genotypes. By incorporating the results obtained from all the above analyses, we propose an updated historical scenario of the formation process of the pantropical distribution of Rhizophora.

\section{Results}

Sequence variability. cpDNA--The aligned sequences of the four cpDNA regions were 3912 bp containing 39 polymorphic sites, which produced 12 haplotypes in 89 samples of Rhizophora (Table 1, Table S1). Five and eight haplotypes were found in the AEP and IWP regions, respectively, and only the haplotype CD was shared between the two regions (Table S2, Fig. 1). The haplotype diversity was higher in IWP species than AEP species regardless of the inclusion or exclusion of $R$. samoensis and the three putative hybrid species from the analyses (Table 2). The nucleotide diversity and theta were higher in IWP species in all including analyses, but these values were similar in excluding analyses of R. samoensis and the putative hybrid species (Table 2).

Ces $A$--The aligned sequences of the Ces $A$ were 555 bp long containing 42 polymorphic sites, which produced 15 alleles in 397 samples of Rhizophora (Table 1, Table S1). Six and ten alleles were found in AEP and IWP species, respectively (Table S2, Fig. 1). The allelic diversity was higher in IWP species, but the nucleotide diversity and theta tended to be higher in AEP species in both analyses (Table 2).

G3pdh--The aligned sequences of the G3pdh were $668 \mathrm{bp}$, there were, however, a ca. $60 \mathrm{bp}$ ambiguously aligned region because of poly $\mathrm{T}$ and $\mathrm{C}$ complex. Therefore, we excluded the complex region for further analysis. As a result, 608 bp was aligned in G3pdh containing 31 polymorphic sites, which produced 14 alleles in 397 samples of Rhizophora (Table 1, Table S1). Seven and eight alleles were found in AEP and IWP species, respectively (Table S2, Fig. 1). The allelic diversity was similar in AEP and IWP species, and the nucleotide diversity was higher in IWP species in both the analyses (Table 2).

Phylogenetic relationships. FindModel identified the best model for each gene: GTR + gamma for cpDNA, Tamura-Nei + gamma for CesA, and Hasegawa-Kishino-Yano + gamma for G3pdh. The results of MP and ML showed congruent topology for the monophyly of each AEP and IWP species except for R. samoensis and $R . \times$ selala (Fig. 1). Rhizophora samoensis had an identical haplotype and allele with AEP species clade, and 


\begin{tabular}{|c|c|c|c|c|c|c|c|}
\hline Taxon & Mangrove region & Oceanic region & Locality & \begin{tabular}{|l|} 
Voucher \\
\end{tabular} & $N_{\mathrm{CP}}$ & $N_{\mathrm{NUC}}$ & Acronyms \\
\hline \multirow{24}{*}{ R. mangle } & \multirow{24}{*}{ AEP } & \multirow{7}{*}{ Pacific } & Panama: El Salado & TK01110601 & 4 & 9 & MPE \\
\hline & & & Ecuador: Jambeli & TK99072003 & 3 & 18 & MEJ \\
\hline & & & Mexico: San Blas & TK03111903 & 1 & 7 & MMS \\
\hline & & & Mexico: Oaxaca & TK03112201 & 1 & 8 & MMO \\
\hline & & & Mexico: Colima & TK08121403 & 1 & 6 & MMM \\
\hline & & & $\begin{array}{l}\begin{array}{l}\text { Costa Rica: Tivives, Pun- } \\
\text { tarenas }\end{array} \\
\end{array}$ & TK07010903 & 1 & 1 & MCT \\
\hline & & & $\begin{array}{l}\text { Costa Rica: Boca del Rio } \\
\text { Damas, Puntarenas }\end{array}$ & TK07011001 & 1 & 7 & MCB \\
\hline & & \multirow{17}{*}{ Atlantic } & Panama: Miramar & TK01110706 & 3 & 9 & MPM \\
\hline & & & Panama: Galeta & TK01103101 & 1 & 14 & MPG \\
\hline & & & $\begin{array}{l}\text { Brazil: Praia do Crispim, } \\
\text { Pará }\end{array}$ & TK99120303 & 3 & 4 & MBP \\
\hline & & & $\begin{array}{l}\text { Brazil: APA de Algodoal, } \\
\text { Pará }\end{array}$ & TK05032501 & 1 & 8 & MBA \\
\hline & & & \begin{tabular}{|l|} 
Brazil: Santa Catarina $2 \mathrm{~km}$ \\
NE from Airport \\
\end{tabular} & TK05031901 & 1 & 4 & MBS \\
\hline & & & $\begin{array}{l}\text { Brazil: Santa Catarina near } \\
\text { the Centro }\end{array}$ & TK05031903 & 2 & 12 & $\mathrm{MBC}$ \\
\hline & & & \begin{tabular}{|l|} 
Brazil: Rio de Janeiro \\
\end{tabular} & TK05032101 & 2 & 11 & MBR \\
\hline & & & Brazil: Pernambuco & KT05032701 & 2 & 4 & MBE \\
\hline & & & U.S.A: Florida & TK05121701 & 2 & 7 & MUF \\
\hline & & & $\begin{array}{l}\text { Mexico: Laguna de Sonte- } \\
\text { comapan, Veracruz }\end{array}$ & TK03112503 & 2 & 4 & MSS \\
\hline & & & $\begin{array}{l}\text { Mexico: Santa Ana, } \\
\text { Veracruz }\end{array}$ & TK03112601 & 2 & 7 & MAL \\
\hline & & & $\begin{array}{l}\text { Mexico: Tonalá river, } \\
\text { Veracruz-Tabasco }\end{array}$ & TK08120906 & 1 & 6 & MML \\
\hline & & & Mexico: Campeche & TK03112405 & 2 & 8 & MMV \\
\hline & & & Costa Rica: Moin, Limon & TK07011201 & 1 & 7 & MMT \\
\hline & & & $\begin{array}{l}\text { Costa Rica: Laguna Gan- } \\
\text { doca, Limon }\end{array}$ & TK07011301 & 1 & 7 & MMC \\
\hline & & & Senegal: South of Mbour & TK00120301 & 2 & 8 & MCM \\
\hline & & & Angola: Luanda & TK01050601 & 2 & 4 & MCL \\
\hline \multirow{5}{*}{ R. racemosa } & \multirow{5}{*}{ AEP } & \multirow{3}{*}{ Pacific } & $\begin{array}{l}\text { Costa Rica: Tivives, Pun- } \\
\text { tarenas }\end{array}$ & TK07010902 & 3 & 16 & RCT \\
\hline & & & \begin{tabular}{|l|} 
Costa Rica: Boca del Rio \\
Damas, Puntarenas \\
\end{tabular} & TK07011002 & 3 & 12 & RCB \\
\hline & & & Ecuador: Esmeraldas & TK02012204 & 5 & 18 & REE \\
\hline & & \multirow{2}{*}{ Atlantic } & Brazil: Mosquiero, Pará & TK05032602 & 2 & 4 & RBM \\
\hline & & & Ghana: Ankobra & KT04092402 & 2 & 11 & RGA \\
\hline \multirow{8}{*}{ R. samoensis } & \multirow{8}{*}{ IWP } & \multirow{8}{*}{ Pacific } & Samoa: Satatoa & TK02102803 & 1 & 9 & SSS \\
\hline & & & Samoa: Safata & TK02102608 & 2 & 7 & SST \\
\hline & & & Tonga: Sopu & TK02102401 & 2 & 7 & STS \\
\hline & & & New Caledonia: Canala & TK07092701 & 1 & 4 & SNC \\
\hline & & & New Caledonia: Yate & TK07092804 & 1 & 7 & SNY \\
\hline & & & New Caledonia: Nouméa & TK07092901 & 1 & 2 & SNN \\
\hline & & & Fiji: Muanikau & KT09012606 & 1 & 4 & SFM \\
\hline & & & Fiji: Vunda Junction & KT09012904 & 1 & 8 & SFV \\
\hline \multirow{5}{*}{ R. stylosa } & \multirow{5}{*}{ IWP } & \multirow{5}{*}{ Pacific } & New Caledonia: Canala & TK07092705 & 2 & 5 & TNC \\
\hline & & & New Caledonia: Yate & TK07092805 & 1 & 8 & TNY \\
\hline & & & New Caledonia: Nouméa & TK07092902 & 1 & 7 & TNN \\
\hline & & & Fiji: Muanikau & KT09012607 & 1 & 4 & TFM \\
\hline & & & Australia: Darwin & TK04121001 & 1 & 2 & TAD \\
\hline \multirow{5}{*}{ R. mucronata } & \multirow{5}{*}{ IWP } & Pacific & Australia: Sarina beach & TK04121502 & 1 & 6 & UAS \\
\hline & & \multirow{4}{*}{ Indian Ocean } & Singapore: Sungei Buloh & TK04121804 & 1 & 4 & USS \\
\hline & & & Seychelles: Port Glaud & KT05090601 & 1 & 4 & USP \\
\hline & & & Mauritius: Mahébourg & KT05090301 & 1 & 5 & UMM \\
\hline & & & South Africa: Beach Wood & KT04091901 & 1 & 5 & UBW \\
\hline
\end{tabular}




\begin{tabular}{|c|c|c|c|c|c|c|c|}
\hline Taxon & Mangrove region & Oceanic region & Locality & Voucher & $N_{\mathrm{CP}}$ & $N_{\mathrm{NUC}}$ & Acronyms \\
\hline \multirow{4}{*}{ R. apiculata } & \multirow{4}{*}{ IWP } & \multirow{2}{*}{ Pacific } & New Caledonia: Canala & TK07092703 & 1 & 6 & ANC \\
\hline & & & Australia: Centenial Lakes & TK04121203 & 1 & 4 & AAC \\
\hline & & \multirow{2}{*}{ Indian Ocean } & Singapore: Sungei Buloh & TK04121803 & 2 & 8 & ASS \\
\hline & & & Sri Lanka: Waikkal & TK04110701 & 1 & 5 & ASW \\
\hline R. $\times$ harrisonii & AEP & Pacific & $\begin{array}{l}\text { Costa Rica: Boca del Rio } \\
\text { Damas, Puntarenas }\end{array}$ & TK07011003 & 2 & 6 & НСB \\
\hline R. $\times$ lamarckii & IWP & Pacific & New Caledonia: Canala & TK07092702 & 1 & 9 & LNC \\
\hline \multirow{3}{*}{ R. $\times$ selala } & \multirow{3}{*}{ IWP } & \multirow{3}{*}{ Pacific } & New Caledonia: Canala & TK07092704 & 1 & 11 & ENC \\
\hline & & & New Caledonia: Yate & TK07092801 & 1 & 1 & ENY \\
\hline & & & Fiji: Lautoka & KT09012902 & 1 & 8 & EFL \\
\hline Bruguiera gymnorrhiza & IWP & Pacific & Australia: Centenial Lakes & TK04121201 & 1 & 1 & BAC \\
\hline \multicolumn{5}{|l|}{ Total } & 90 & 398 & \\
\hline
\end{tabular}

Table 1. Localities of sampled populations of Rhizophora and Bruguiera species and sample size for chloroplast $\left(N_{\mathrm{CP}}\right)$ and nuclear DNA $\left(N_{\mathrm{NUC}}\right)$ analyses.

R. $\times$ selala had the same cpDNA haplotype with AEP species and same nuclear alleles with the AEP and IWP clades in hetero. Rhizophora mangle and $R$. racemosa G. Mey. did not make its own clade within the AEP clade. Rhizophora apiculata Blume formed a monophyletic clade with $R . \times$ lamarckii Montrouz., and $R$. stylosa Griff. and $R$. mucronata Lam. formed a monophyletic clade together in the IWP clade.

Minimum spanning network of haplotypes. All three Minimum Spanning Networks constructed using haplotypes of cpDNA, CesA, and G3pdh showed clear separation of IWA and AEP groups (Fig. S2). A haplotype possessed by Rhizophora species (colored in orange: GH in cpDNA, F in CesA, G in G3pdh) was found only from the Atlantic region and was placed at the basal position of the AEP group in each network.

Global population genetic structure. Global distribution of cpDNA haplotype and nuclear allele in the six non-putative hybrid species are shown in Fig. 1. The details of haplotype and allele frequency are in Table S2. The patterns of nuclear alleles in putative hybrid species were shown in Table 3 and Table S3. There are only one cpDNA haplotype (CD) and CesA and G3pdh alleles (both B) which can be observed in both AEP and IWP species. These haplotypes and alleles were commonly and geographically-widely found in the species in the AEP region; however, they were found only in $R$. samoensis and $R . \times$ selala in the IWP region.

In the AEP region, each haplotype and allele were widely distributed. A remarkable genetic differentiation between the Pacific and the Atlantic side were found in cpDNA haplotypes, although there are few overlaps between the two regions in terms of both nuclear DNA alleles. Populations on the Pacific coast in Mexico and Costa Rica had the alleles which widely dominant in the Atlantic region. In the IWP region, there are several haplotypes and alleles which were widely distributed or unique to a single locality.

Divergence times. To estimate divergence time for deeper nodes within Rhizophora, we conducted phylogenetic analyses with two selected cpDNA regions ( $r b c L$ and $a t p B-r b c L$ intergenic region) and two nuclear genes combining with the available data set deposited in DDBJ (DNA Data Bank of Japan). The most recent common ancestor of Rhizophora and sister mangrove genera, Ceriops Arn. and Kandelia (DC.) Wight et Arnott, was estimated to be 36.0 Ma (95\% HPD 34.5-38.2 Ma: Fig. 2) by using cpDNA data including 27 OTUs gathered in the Bayesian analysis with the two calibration points. The split between AEP and IWP clades of Rhizophora was estimated to be $10.6 \mathrm{Ma}(5.4-16.3 \mathrm{Ma})$. In the AEP clade, the split between $R$. racemosa and $R$. mangle/R. racemosa/R. samoensis clades were estimated to be $3.0 \mathrm{Ma}(0.5-6.0 \mathrm{Ma})$ in the cpDNA analysis. In the IWP clade, the split between $R$. apiculata and $R$. stylosa/R. mucronata clades were estimated to be $7.4 \mathrm{Ma}(3.1-12.2 \mathrm{Ma})$. The estimation time of the split between AEP and IWP clades estimated using the first calibration point only was 10.3 - 34.0 Ma $(2.2-53.7 \mathrm{Ma})$ depending on the data set (Table S4).

Biogeographic inference. Biogeographic stochastic mapping analyses in BioGeoBEARS yielded the BAYAREA + J model as the best- fitting model for the phylogeny of all Rhizophoraceae species (Table S5). The ancestral ranges of IWP and AEP were Indo-Malesia and Australasia $(\mathrm{EF})(\mathrm{ML}$ probability $=0.60)$ and East America and West Africa $(\mathrm{BC})(\mathrm{ML}$ probability $=0.26)($ Fig. 2). The estimated ancestral range of all AEP species was East America and West Africa (BC) (ML probability=0.92) (Fig. 2).

Hybridization patterns in the South Pacific Islands. The results of PCR-RFLP showed that all individuals of $R$. stylosa and $R$. samoensis including their hypocotyls possessed their specific genotypes in cpDNA and nuclear DNA, and all individuals of $R . \times$ selala possessed a particular combination of cpDNA haplotype, $R$. samoensis genotype, and nuclear DNA genotypes, $R$. stylosa and $R$. samoensis in hetero, which can be expected of F1 hybrid genotype (Tables S2 \& S3). All propagules had the same genotypes as their mother tree of $R$. stylosa or R. samoensis. The results of STRUCTURE based on microsatellite data were shown in Fig. 3 and Fig. S1. The 
value of log-probability began to plateau at $K=4$ and the highest delta $K$ value was returned at $K=2$ so that we showed $K=2$ and $K=4$ as most probable numbers of the cluster. In $K=2$, the two clusters represented $R$. stylosa and $R$. samoensis, respectively, and both clusters can be admixed fifty-fifty in three populations of $R . \times$ selala. In $K=4$, each of the two clusters represented $R$. stylosa or R. samoensis mostly depending on the locality. Red and green clusters were found in $R$. stylosa from New Caledonia or Fiji, and blue and orange ones in R. samoensis. Two clusters found in each locality were admixed in $R . \times$ selala.

\section{Discussion}

Phylogeny and timing of diversification of Rhizophora. This study provided the most comprehensive phylogeny that explains the present pantropical distribution of a mangrove genus Rhizophora. We included distinct lineages from a wide range of the AEP region and showed that all AEP samples were in the AEP clade that is sister to the IWP clade in all analyses using multiple DNA markers (Fig. 1). Although trans-oceanic dispersal and frequent hybridization have been reported in the genus Rhizophora, this result ensures an adequate discussion of the divergence time between the AEP and IWP lineages of Rhizophora based on the phylogenetic tree.

The divergence time between the AEP and IWP lineages was $10.6 \mathrm{Ma}$ by using cpDNA and broad outgroup taxa (Fig. 2) and 11.0-11.5 Ma by a combination of three different regions (cpDNA, CesA, and G3pdh) (Table S4) which are similar to the ones, $12.7 \mathrm{Ma}$ by five nuclear genes ${ }^{15}$ and $10.8 \mathrm{Ma}$ by 590 coding genes ${ }^{16}$ and far different from $48( \pm 3)$ Ma by cpDNA and ITS $^{14}$. Because our study based on global sampling showed the monophyly of the AEP lineage and similar estimates for the divergence time between the IWP and AEP linages, we consider that the estimates of Xu et al. ${ }^{16}$ also can be reasonable even though they used only one sample from the AEP region.

The separation of the AEP and IWP lineages reasonably coincide with the closure of the Tethys Seaway. The biogeographic stochastic mapping analyses suggested that the common ancestor of the AEP and IWP lineages was separated into two ancestral ranges 10.6 Ma: the Atlantic region (BC) for the AEP lineage, and the IndoPacific region (E, F, and EF) for the IWP linage (Fig. 2). The separation can be attributed to the formation of the land barrier at Tethys Seaway that separated the Atlantic and Indo-Pacific regions around 11 to $20 \mathrm{Ma}^{20-24}$. The common ancestor of the AEP lineage at the Atlantic (BC) was suggested to expand the range to the Pacific regions (A and $\mathrm{ABCF}$ ) in the later diversification (Fig. 2). Although there were only three extant AEP lineages of Rhizophora in the dated tree (Fig. 2) due to less length of sequence data, longer length of cpDNA sequence showed the presence of five haplotypes (AB, CD, E, F, and GH) (Fig. 1a, Fig. S2), and three haplotypes (CD, E, and F) were exclusively found in the Pacific region (Fig. 1a,d). This remarkable segregation between the Pacific and Atlantic populations can be attributed to the closure of the Isthmus of Panama at $3 \mathrm{Ma}^{25}$ after the range expansion of Rhizophora species from Atlantic to the Pacific.

Our results provided clear evidence of the recent trans-pacific migration of $R$. mangle from the Pacific side of the New World (AEP) to the South Pacific Islands (IWP) $)^{13,14}$. The haplotype possessed by $R$. samoensis from the South Pacific Islands and $R$. mangle from the Pacific side of the New World was identical both in cpDNA (CD) and nuclear DNA markers (B in both markers) (Fig. 1). Our data also confirmed that $R . \times$ selala in the South Pacific Islands was a F1 hybrid asymmetrically formed by $R$. stylosa as father lineage and $R$. samoensis as mother lineage. In addition, our microsatellite data suggested that independent events of hybrid formation occurred recurrently in New Caledonia and Fiji (Fig. 3). These results suggest that $R . \times$ selala are F1 hybrids between the species from IWP and AEP lineages. Although hybrids are recurrently formed when the two lineages meet, they are sterile F1 hybrids because of strong reproductive isolation caused by long separation of parental lineages for about $11 \mathrm{Ma}$. Our results supported the previous observation that $R . \times$ selala had low pollen fertility and abnormal tetrads ${ }^{18,19}$.

Updated scenario for the formation of the distribution pattern of Rhizophora. According to our findings and some previous studies, we finally updated the historical scenario behind the formation of the distribution pattern of the mangrove genus Rhizophora, proposed by Duke et al. ${ }^{5}$ We illustrated a synthetic scenario of the historical process with the tectonic events and changes of global temperature that shape the present pantropical distribution of the mangrove genus Rhizophora in Fig. 4 and as followings:

Eocene-Oligocene (50-23 Ma). Rhizophora originated during the Eocene and was distributed worldwide by the Middle and Late Eocene evidenced by several comprehensive assessments of fossil records ${ }^{6,7,26-29}$. Our biogeographic analysis supported that the origins of extant Rhizophora were mostly in IWP region and further spread to AEP region (Fig. 2). The mangrove habitat might have been more widely extended in longitude than at present, because the average temperature of the earth was $4-12{ }^{\circ} \mathrm{C}$ higher ${ }^{30}$. In fact, the pollen of mangrove palm, Nypa, was reported from the Early Eocene layer in Tasmania ${ }^{31}$ and New Zealand ${ }^{32}$, where no mangrove habitat occurs at present. The wider longitudinal distribution area of mangrove might be achieved by the migration of propagules in the east-west direction between the Old World and New World, via the southern route around the African continent. Furthermore, ocean currents through "Tethys Seaways (Mediterranean Sea)" also could work as a dispersal corridor between the two regions (Fig. 4, "Globalization of Rhizophora").

Miocene (23-10 Ma). By geological approaches, the closure of Tethys Seaways was estimated from 11 to $20 \mathrm{Ma}^{20-24}$ which is consistent with our estimation of the divergence time ca. $11 \mathrm{Ma}$ for the deep phylogenetic break between IWP and AEP lineages. The closure of the Tethys Seaway created a land barrier between the IWP and AEP regions ${ }^{33}$. In addition, Mid-Miocene cooling occurred at the same time ${ }^{30}$ would narrower the distribution range to the equator and prevented the migration of propagules via the southern route around the African continent (Fig. 4, "IWP \& AEP separation"). 
Figure 1. Trees of maximum parsimony topologies and geographical distributions of the haplotype/allele from combined chloroplast DNA sequences (a), and nuclear DNA sequences (b, CesA and c, G3pdh) of Rhizophora species. The ML analysis yield congruent MP topology (not shown). Numbers above branches indicate bootstrap probabilities greater than $50 \%$ for parsimony (left) and likelihood (right), respectively. Colored circles on the right of OTU indicate DNA sequences shown on the maps (d-f) indicating localities of sampled populations. Colored pie chart on the map indicate the frequency of chloroplast DNA haplotypes (d), and nuclear DNA alleles (e, CesA and f, G3pdh) of Rhizophora species except for hybrid species. Abbreviations in brackets in the tree and along pie chart on the map indicate species names: man, $R$. mangle; rac, $R$. racemosa; sam, $R$. samoensis; sty, R. stylosa; api, R. apiculata; muc, $R$. mucronata; $\times$ ha, $R . \times$ harrisonii, $\times$ la, $R . \times$ lamarckii, $\times$ se, $R . \times$ selala.

Late Miocene to Present (10 Ma-). The global cooling at Late Miocene and Pleistocene might have promoted a decrease in the population size for many mangrove species via sea level drop, and separation to regional populations may lead allopatric speciation in Rhizophora. In the AEP region, both R. mangle and R. racemosa expanded their distribution across the American continents from the Atlantic region via migration through the Panama Seaway. After the closure of the Panama Seaway at $3 \mathrm{Ma}^{25}$, genetic differentiation between the Pacific and Atlantic populations of both species occurred. In the IWP region, the R. apiculate, $R$. stylosa, and $R$. mucronata might form their present distribution through different demographic histories in each species despite having sympatric distributions today ${ }^{34,35}$. Finally, eventual trans-Pacific dispersal of $R$. mangle happened from American continents to the South Pacific. The extremely long-distance dispersal caused secondary contact between distinct lineages of Rhizophora, which can independently produce the sterile F1 offspring in the South Pacific Islands (Fig. 4, "Trans-Pacific dispersal from AEP to IWP").

\section{Methods}

Plant collections. We collected leaf samples from 56 populations of all eight species including three putative hybrid species across the IWP and AEP regions for phylogenetic analyses (Table 1). Upon collection, leaf samples for DNA extraction were put in reclosable plastic bags and dried with silica gel. We also collected 15 populations of $R$. stylosa, $R$. samoensis, and $R . \times$ selala in New Caledonia and Fiji for investigation of hybridization pattern in the South Pacific Islands (Table 3). In addition, a part of the hypocotyl of propagule for DNA extraction was also collected from R. samoensis and R. stylosa in Fiji (Table 3). Twenty-nine populations from the AEP region and nine populations form the IWP region using in this study are the same as those used in a previous study ${ }^{13}$. Identification of the species was done following the keys in Tomlinson ${ }^{10}$ and at least one voucher specimen from each population and species was deposited in the Herbarium at the Herbarium of University of Ryukyus (URO).

DNA extraction and sequencing. Genomic DNA was extracted from the dried leaf and hypocotyl tissue using the cetyltrimthyl ammonium bromide (CTAB) extraction method ${ }^{36}$ and purified using GENECLEAN III Kit (MP Biomedicals, Solon, USA). PCR amplification and sequencing were performed using 1 to 18 individuals from each population (Table 1). Sequences of four cpDNA regions, atpB-rbcL intergenic spacer (IGS), $\operatorname{trnS}$ - $\operatorname{trn} G$, rpl16 intron, and $r b c L$ gene, were determined with the same protocol as in the previous study ${ }^{13}$. Other available sequences from Rhizophoraceae were obtained from GenBank accessions (Table S1). In addition, a part of rpl16 intron sequence was determined in 165 individuals of $R$. stylosa, R. samoensis, and R. $\times$ selala, and 64 hypocotyls of the propagule of $R$. stylosa and $R$. samoensis in New Caledonia and Fiji. Two nuclear DNA regions, glyceraldehyde 3 phosphate dehydrogenase $(G 3 P D H)$, and cellulose synthase (CesA) homolog were amplified using primers reported in Strand et al. ${ }^{37}$ and Cronn et al. ${ }^{38}$. Sequencing reaction was performed with using primers GPDX7F $^{37}$ and G3PDH-788R1 (5'-CAATGAAGTCTGTGGATACCAA-3') for G3PDH, and CesA-1150F (5'CCACCTGAGCAGCAGATGGAAG-3') and CesA-1800R (5'-ACGACAGTTGAAAGTGGCTGTGC-3') for CesA. In cases where the phenogram of the two nuclear DNA regions showed duplicated peaks in more than two sites, we segregate the alleles of the PCR amplicon with single-strand conformation polymorphism (SSCP) analyses $^{39}$ and re-sequenced after acrylamide gel extraction.

Phylogenetic inference. Genetic diversity parameters were estimated using DnaSP $5.10^{40}$. Phylogenetic analyses were performed using the Wagner maximum parsimony (MP) and Maximum Likelihood (ML) methods based on three different matrices, the combined sequence data of four cpDNA, G3PDH and C3esA, separately. Bruguiera gymnorrhiza was used as outgroup for all of the Rhizophora species according to previous phylogenetic study ${ }^{11}$. MP and ML analyses were conducted using PAUP ${ }^{\star 41}$ and RAxML ${ }^{42}$, respectively. For the ML analyses, FindModel (http://hcv.lanl.gov/content/hcvdb/findmodel/) was used to find the best fitting model for nucleotide sequence evolution.

Minimum spanning network. We used PopART ${ }^{43}$ to construct a minimum spanning network ${ }^{44}$ using the alignments of haplotype and allele sequences of cpDNA, G3PDH and C3esA.

Divergence time estimates. To test temporal aspects of phylogeographic breaks, we inferred divergence times using a relaxed-clock method in the program BEAST v1.6.2 $2^{45}$. Two chloroplast regions, $r b c L$ and atpB$r b c L$, were used to conduct comprehensive analyses by including compatible data from other genera in Rhizophoraceae in previous phylogenetic study ${ }^{11}$. In addition, two nuclear regions, CesA and G3pdh obtained in this study were used for the dating analyses. We estimated divergent times using 12 different combinations of data 


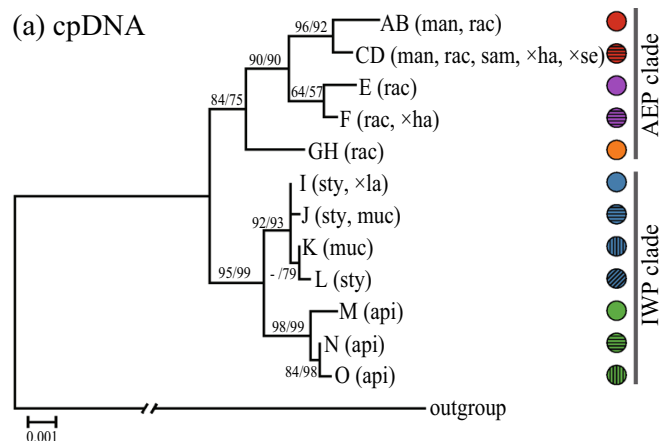

(b) CesA
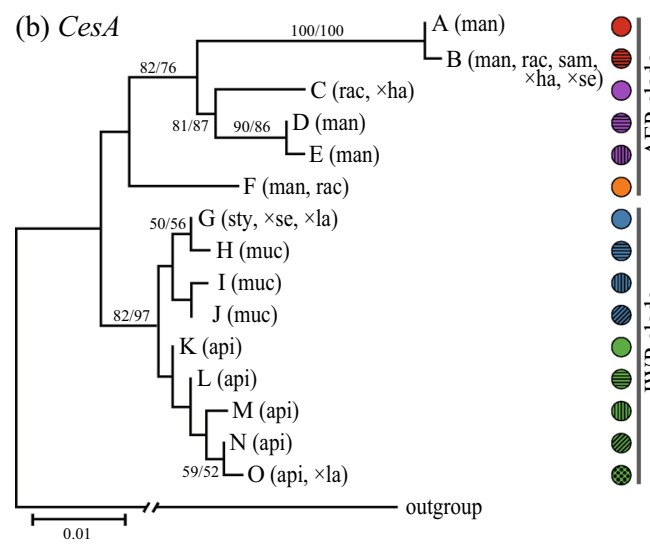

(c) G3pdh
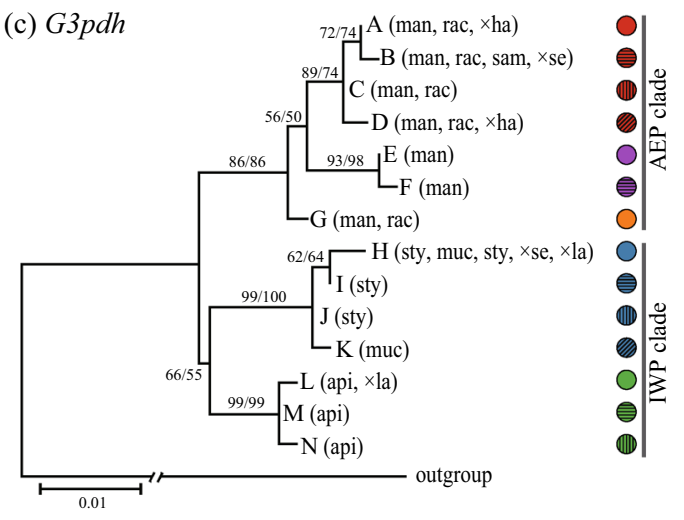

(d) cpDNA

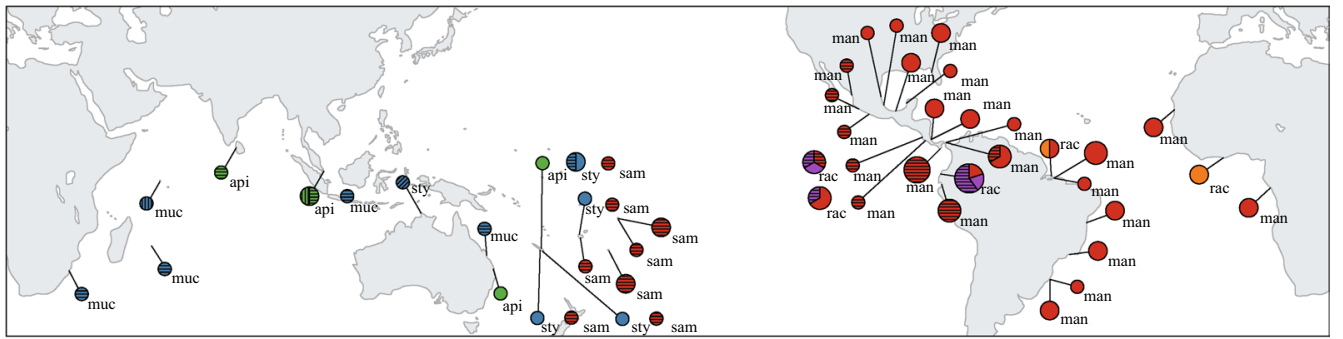

(e) $\operatorname{Ces} A$

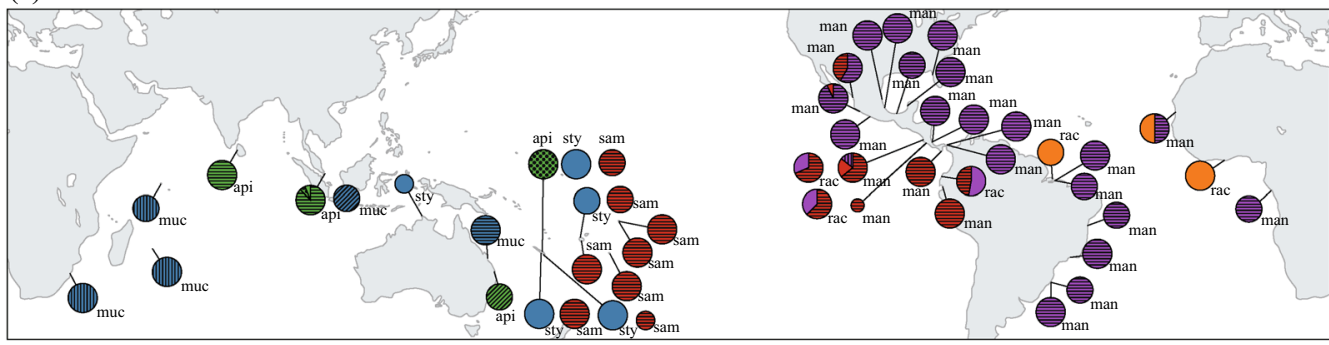

(f) G3pdh

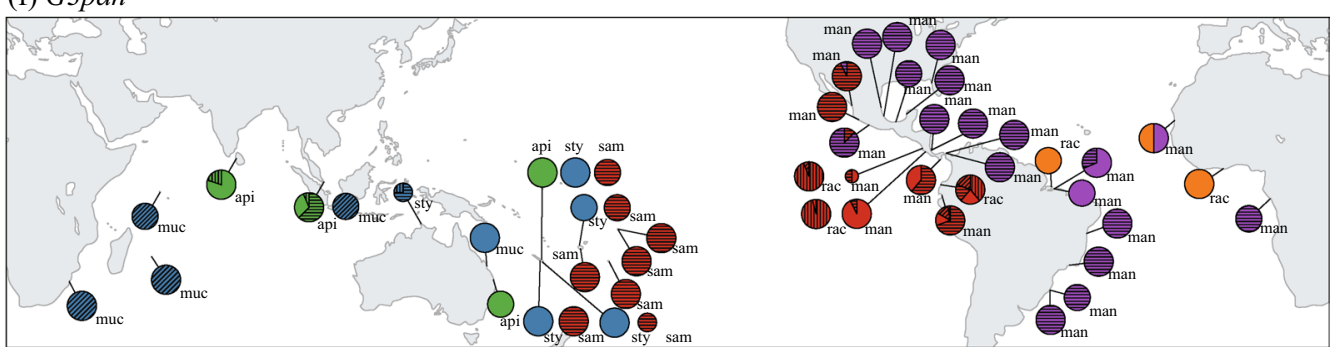




\begin{tabular}{|c|c|c|c|c|c|c|c|c|c|c|c|c|c|c|c|c|}
\hline \multirow[b]{2}{*}{ Taxon } & \multicolumn{6}{|c|}{ cpDNA } & \multicolumn{5}{|c|}{ CesA } & \multicolumn{5}{|c|}{ G3pdh } \\
\hline & $N_{\mathrm{P}}$ & $N$ & $H$ & $H d$ & $P i$ & $\theta$ & $N$ & $H$ & $H d$ & $P i$ & $\theta$ & $N$ & $H$ & $H d$ & $P i$ & $\theta$ \\
\hline R. mangle & 24 & 42 & 2 & 0.438 & 0.0009 & 0.0005 & 180 & 5 & 0.375 & 0.0122 & 0.0078 & 180 & 7 & 0.556 & 0.0054 & 0.0030 \\
\hline R. racemosa & 5 & 15 & 5 & 0.829 & 0.0027 & 0.0021 & 61 & 3 & 0.652 & 0.0222 & 0.0094 & 61 & 5 & 0.629 & 0.0032 & 0.0021 \\
\hline R. samoensis & 8 & 10 & 1 & 0.000 & 0.0000 & 0.0000 & 48 & 1 & 0.000 & 0.0000 & 0.0000 & 48 & 1 & 0.000 & 0.0000 & 0.0000 \\
\hline R. stylosa & 5 & 6 & 3 & 0.600 & 0.0003 & 0.0004 & 26 & 1 & 0.000 & 0.0000 & 0.0000 & 26 & 3 & 0.147 & 0.0006 & 0.0010 \\
\hline R. mucronata & 5 & 5 & 2 & 0.400 & 0.0001 & 0.0001 & 24 & 3 & 0.582 & 0.0023 & 0.0012 & 24 & 2 & 0.383 & 0.0024 & 0.0014 \\
\hline R. apiculata & 4 & 5 & 3 & 0.800 & 0.0008 & 0.0007 & 23 & 5 & 0.766 & 0.0051 & 0.0024 & 23 & 3 & 0.426 & 0.0020 & 0.0014 \\
\hline$R . \times$ harrisonii & 1 & 2 & 2 & 1.000 & 0.0033 & 0.0033 & 6 & 2 & 0.485 & 0.0156 & 0.0106 & 6 & 2 & 0.530 & 0.0085 & 0.0053 \\
\hline R. $\times$ lamarckii & 1 & 1 & 1 & 0.000 & 0.0000 & 0.0000 & 9 & 2 & 0.529 & 0.0047 & 0.0026 & 9 & 2 & 0.529 & 0.0099 & 0.0054 \\
\hline$R . \times$ selala & 1 & 3 & 1 & 0.000 & 0.0000 & 0.0000 & 20 & 2 & 0.513 & 0.0192 & 0.0088 & 20 & 2 & 0.513 & 0.0156 & 0.0071 \\
\hline AEP & 30 & 59 & 5 & 0.655 & 0.0014 & 0.0015 & 247 & 6 & 0.604 & 0.0181 & 0.00851 & 247 & 7 & 0.728 & 0.0064 & 0.00283 \\
\hline IWP & 24 & 30 & 8 & 0.733 & 0.0039 & 0.0026 & 150 & 8 & 0.751 & 0.0199 & 0.00741 & 150 & 8 & 0.729 & 0.0162 & 0.00637 \\
\hline All & 54 & 89 & 12 & 0.763 & 0.0029 & 0.0024 & 397 & 13 & 0.763 & 0.0237 & 0.01045 & 397 & 14 & 0.830 & 0.0134 & 0.00684 \\
\hline $\mathrm{AEP}^{*}$ & 29 & 57 & 5 & 0.643 & 0.0014 & 0.0015 & 241 & 6 & 0.595 & 0.0178 & 0.0085 & 241 & 7 & 0.720 & 0.0064 & 0.0028 \\
\hline IWP $^{*}$ & 14 & 16 & 7 & 0.750 & 0.0015 & 0.0013 & 73 & 7 & 0.769 & 0.0045 & 0.0023 & 73 & 7 & 0.717 & 0.0094 & 0.0045 \\
\hline All $^{*}$ & 43 & 73 & 12 & 0.763 & 0.0029 & 0.0025 & 314 & 13 & 0.749 & 0.0222 & 0.0108 & 314 & 14 & 0.820 & 0.0129 & 0.0071 \\
\hline
\end{tabular}

Table 2. Genetic diversity parameters estimated by chloroplast and nuclear DNA sequences. $N_{\mathrm{p}}$ number of populations; $N$, number of individuals; $H$, number of haplotypes or alleles, $H d$, haplotype (allele) diversity; $P i$, nucleotide diversity; $\theta$, number of mutation per site.

\begin{tabular}{|l|l|l|l|}
\hline Locality & Taxon & Voucher & N \\
\hline \multirow{4}{*}{ New Caledonia: Canala } & R. stylosa & TK07092705 & 8 \\
\cline { 2 - 4 } & R. $\times$ selala & TK07092704 & 8 \\
\cline { 2 - 4 } & R. samoensis & TK07092701 & 7 \\
\hline \multirow{3}{*}{ New Caledonia: Yate } & R. stylosa & TK07092805 & 15 \\
\cline { 2 - 4 } & R. $\times$ selala & TK07092801 & 8 \\
\cline { 2 - 4 } & R. samoensis & TK07092804 & 10 \\
\hline \multirow{3}{*}{ New Caledonia: Nouméa } & R. stylosa & TK07092902 & 16 \\
\cline { 2 - 4 } & R. samoensis & TK07092901 & 5 \\
\hline \multirow{2}{*}{ Fiji: Muanikau* } & R. stylosa & TK09012607 & 6 \\
\hline & R. samoensis & KT09012606 & 17 \\
\hline \multirow{2}{*}{ Fiji: Bau } & R. stylosa & TK09012702 & 8 \\
\hline & R. samoensis & KT09012701 & 22 \\
\hline \multirow{3}{*}{ Fiji: Lautoka } & R. stylosa & TK09012903 & 19 \\
\cline { 2 - 4 } & R. $\times$ selala & KT09012902 & 1 \\
\hline & R. samoensis & KT09012901 & 15 \\
\hline Total & \multicolumn{2}{|l}{} & 165 \\
\hline
\end{tabular}

Table 3. Localities of sampled populations of Rhizophora samoensis, R. stylosa, and R. $\times$ selala in New Caledonia and Fiji, and sample size for PCR-RFLP and microsatellite analyses. ${ }^{*} 34$ and 30 hypocotyls were collected in R. samoensis and R. stylosa, respectively.

and taxon because the nucleotide diversity and available sample of the outgroup taxa were different depending on the DNA regions. As previously reported ${ }^{13,14}$, Rhizophora species had experienced hybridization and shared haplotype/genotype partially, hence the sorting of haplotypes/genotypes into a sample increased the number of OTUs which shared identical haplotype or genotype. We first conducted a multispecies coalescent analysis ${ }^{46}$ in the dating analyses using the combination of different genes. The multispecies coalescent analysis estimates a species (taxon) tree based on unlinked multi-locus sequence data, taking into account that gene trees are incorporated in a shared species (taxon) tree by following the stochastic coalescent process. For the analysis that included multiple regions, three OTUs from Rhizophora samples, such as R. stylosa-R. mucronata, R. apiculata, and $R$. mangle $-R$. racemosa were used for the coalescent analysis. The analysis was conducted using the program BEAST v1.6.2 $2^{45}$. We used the fossil record to place priors on the crown age of a node, arising of mangrove clade (Bruguiera Lam., Kandelia, Ceriops, and Rhizophora) in Rhizophoraceae. In the review of mangrove fossil record $^{7}$, fruits and pollen of ancestors very close to modern mangrove genera in Rhizophoraceae existed in the 


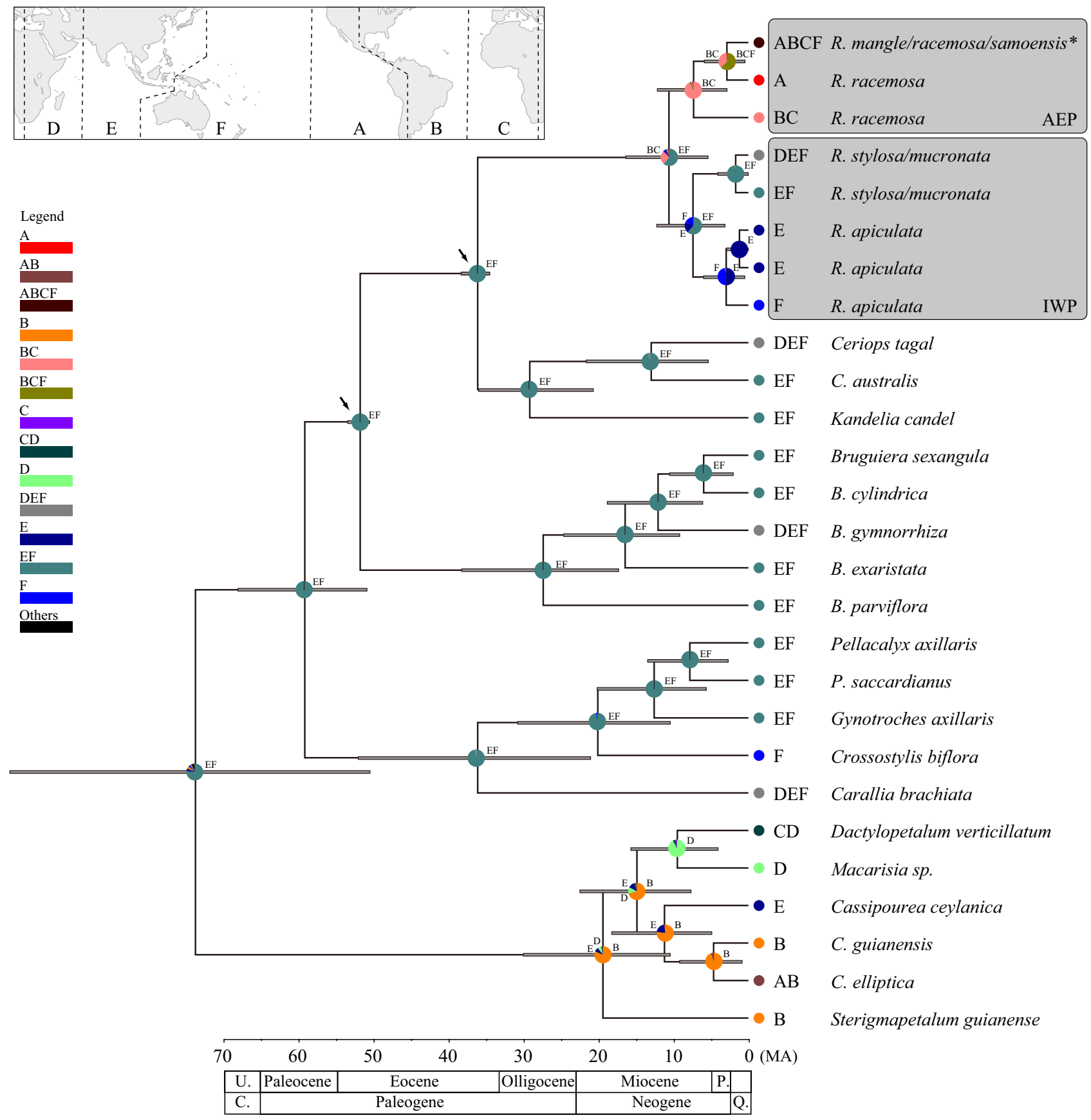

Figure 2. Chronograph and biogeographic history for Rhizophora and outgroup taxa based on the relaxedclock Bayesian MCMC methods in BEAST and BioGeoBEARS in RASP using chloroplast DNA sequences. Error bars on nodes indicate $95 \%$ highest posterior densities around the mean dates. Arrows indicate fossil calibration points described in Methods. Probability of ancestral states are shown in pie chart. * Rhizophora samoensis is distributed in the IWP region but has an identical haplotype with AEP species.

Early Eocene (50 Ma), but not in the Palaeocene (55 Ma) when the fossil record of another mangrove genus, Nypa, is abundant worldwide. Therefore, we set a lognormal prior offset of $50 \mathrm{Ma}$ with a mean and standard deviation of 0.5 for the first calibration point. In addition, ancestral Rhizophora fossil has been reported to the late Eocene (33.9-38 Mya) ${ }^{47}$. We set a lognormal prior offset of $34 \mathrm{Ma}$ with a mean and standard deviation of 0.5 for crown age of a node of a monophyly clade of Ceriops, Kandelia, and Rhizophora for the second calibration point. We used the two calibration points for the cpDNA analyses using broad species in Rhizophoraceae. On the other hand, we only used the first calibration point for 12 different combinations of data and taxon due to the limitation of the taxon coverage to use the second calibration point. We conducted three independent searches of $10 \times 10^{7}$ generations sampling every 1000 generations under the models selected by FindModel in each genetic region. The results log files from the three runs were combined by the program LogCombiner v1.7.5 ${ }^{45}$. We confirmed that ESS values of all estimated parameters after $20 \%$ burn-in were $>200$ in the program Tracer v1.5 $5^{45}$. We 


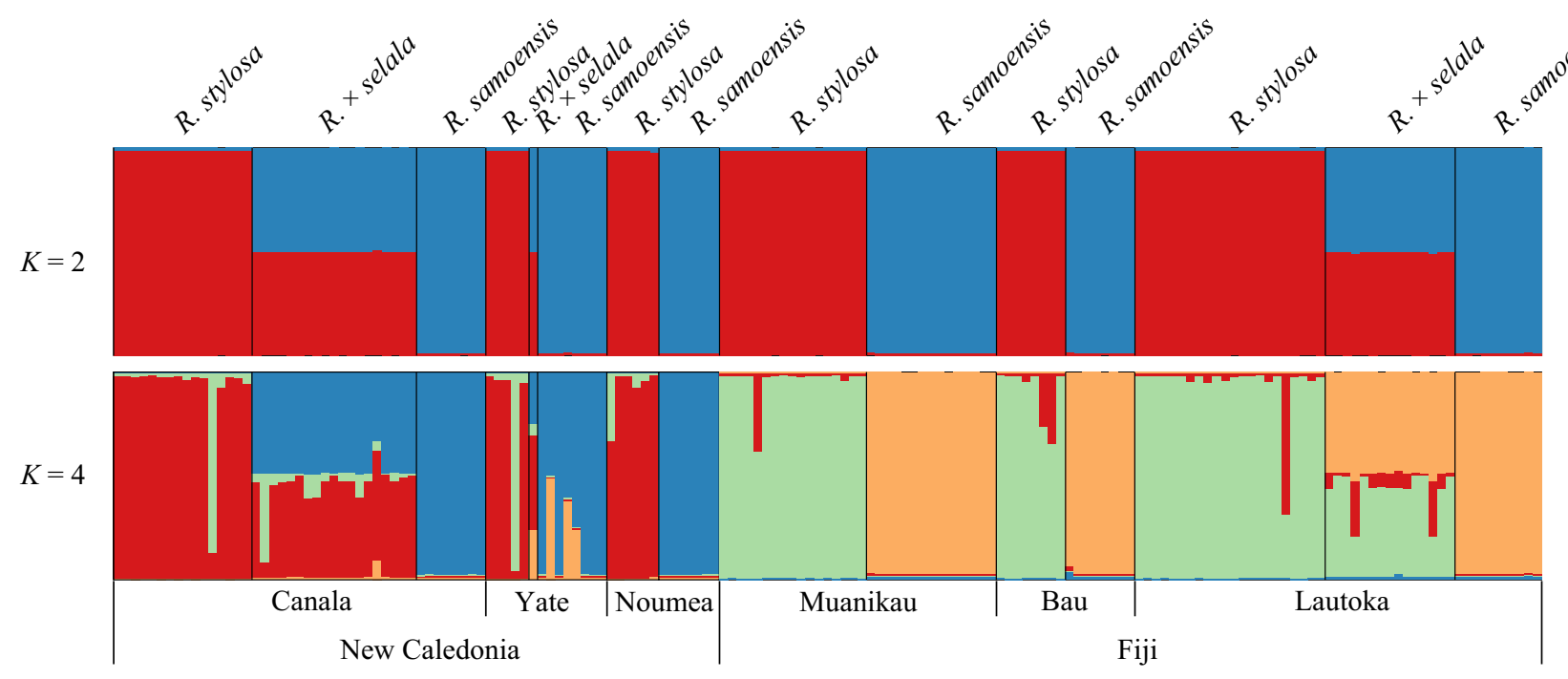

Figure 3. Results of STRUCTURE of Rhizophora species in the South Pacific Islands. Vertical columns represent individual plants, and the heights of bars of each color are proportional to the posterior means of estimated admixture proportions. The number of cluster $K=2$ and $K=4$ are shown.

(Ma) Geochronology Tectonic events Global temperature Phylogeny

$\begin{aligned} 0 & \text { Pleistocene } \\ 2.4 & \text { Pliocene }\end{aligned}$

23

Oligocene

35

56

Paleocene

65

Miocene
Isthmus of Panama formation (3)

Australia collided with Asia

$\begin{array}{ll}\text { India collided } \\ \text { Eocene } & \text { with Asia (40) }\end{array}$

Australia \&

South America separated (45)

Australia \&

Antarctica separated (30)

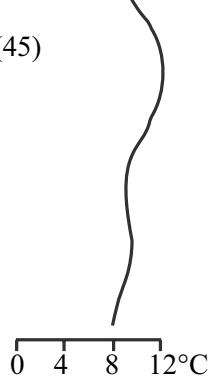

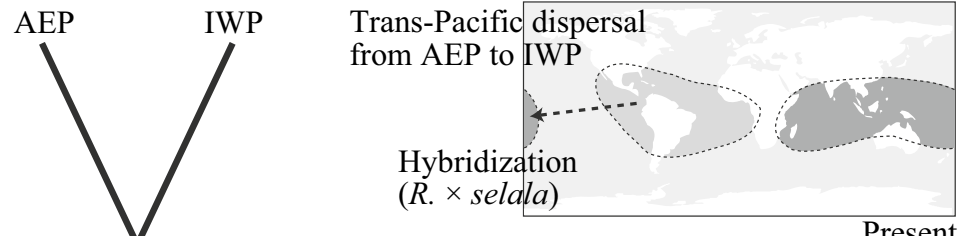

AEP \& IWP

separation (ca. $11 \mathrm{Ma}$ )
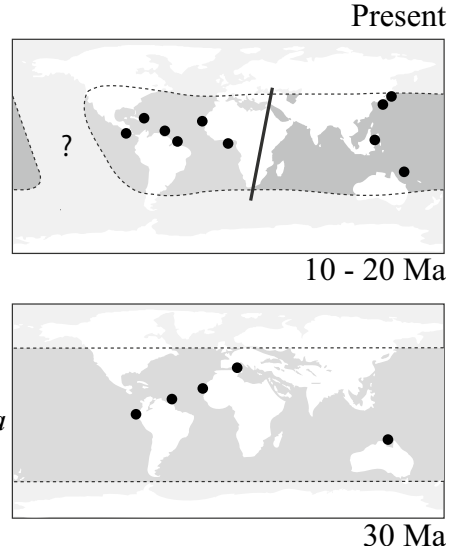

Globalization of Rhizophora

Rhizophora fossil

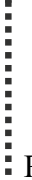

Rhizophoraceae fossil

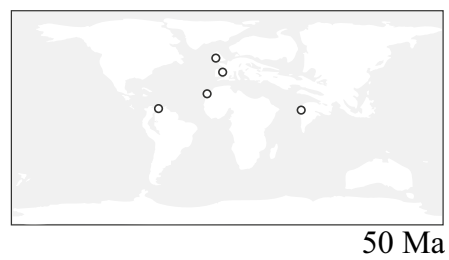

ofossil of Rhizophoraceae - fossil of Rhizophora

Figure 4. Historical scenario of diversification of Rhizophora. Concurrent significant tectonic events, global temperature, and fossil occurrences have been listed for comparison with the result of phylogenetic analysis in this study. The patterns of global temperature and fossil occurrences were modified from previous studies ${ }^{7,30}$. 
also generate a maximum clade credibility tree using $20 \%$ burn-in trees obtained in three runs by LogCombiner v1.7.5 and TreeAnnotator v1.7.5 $5^{45}$ and presented the mean and 95\% highest posterior density (HPD) of node ages by FigTree v1.4 $4^{48}$.

Biogeographic inference. To infer the ancestral range for Rhizophora, we used the ML method implement in BioGeoBEARS $0.2 .1^{49}$ in the RASP 4.2 package $^{50}$. We defined the geographical ranges as A= West America, $\mathrm{B}=$ East America, $\mathrm{C}=$ West Africa, $\mathrm{D}=$ East Africa, $\mathrm{E}=$ Ind-Malesia, and $\mathrm{F}=$ Australasia according to the previously reported definitions of geographic ranges of Rhizophora ${ }^{5}$. We estimated ancestral ranges and biogeographical events on the BEAST MCC trees of Rhizophoraceae species based on two cpDNA regions (Fig. 2), using a likelihood-based framework in BioGeoBEARS that allows for testing various biogeographical models. The best model was selected using AIC corrected for sample size (AICc). We rerun the BioGeoBEARS analysis on 100 randomly sampled post-burn- in BEAST chronograms. The number of maximum areas per ancestral range was set to four.

PCR-RFLP and microsatellite analysis. To reveal hybridization patterns in the South Pacific Island, PCR-RFLP and microsatellite genotyping were conducted in 165 individuals of $R . \times$ selala, R. samoensis, and R. stylosa in New Caledonia and Fiji, and 64 hypocotyls of propagule of R. samoensis and R. stylosa in Fiji (PCR-RFLP only). For PCR-RFLP, the amplified G3PDH and CesA fragments in the 165 individuals and 64 hypocotyls were digested with restriction enzymes, MspI (CCGG) and Sau3A (GATC), respectively. Based on the sequence data, we expected the length of DNA fragments of $R$. stylosa after this treatment to be ca. 10, 30, $670 \mathrm{bp}$ in G3PDH and 50, 50, 70, $390 \mathrm{bp}$ in CesA, and those of $R$. samoensis to be 10, $700 \mathrm{bp}$ and 50, 70, $440 \mathrm{bp}$. Digested DNA fragments were detected $2 \%$ agarose electrophoresis, and determined genotypes of two nuclear DNA regions. In addition, four microsatellite markers (RM50, RS19, RS33, and RS59) developed by Takayama et al. ${ }^{51,52}$ were selected for the analysis of hybridization patterns in New Caledonia and Fiji, because the four markers can be constantly amplified in both AEP and IWP species. We determined microsatellite genotypes in 165 individuals of $R . \times$ selala, $R$. samoensis, and R. stylosa in New Caledonia and Fiji. PCR amplification and genotyping were performed according to the previous study ${ }^{13}$. Genetic structure was evaluated by the Bayesian clustering method using STRUCTURE 2.3.3 ${ }^{53-55}$. Markov chain Monte Carlo (MCMC) searches consisted of 100,000 "burn-in" steps, followed by 100,000 iterations. Twenty replicate runs were performed at each $K$ from 1 to 10 and calculated the log-probability and the delta $K$ proposed by Evanno et al. ${ }^{56}$, given a certain value of $K$ for seeking the best-fit $K$ value for the data.

\section{Data availability}

All data sets are provided in the Supplementary Information and deposited in DDBJ.

Received: 9 October 2019; Accepted: 5 March 2021

Published online: 30 March 2021

\section{References}

1. Spalding, M., Kainuma, M. \& Collins, L. World Atlas of Mangroves. (Earthscan, 2010).

2. Duke, N. et al. A world without mangroves?. Science 317, 41-42 (2007).

3. Friess, D. et al. The state of the world's mangrove forests: past, present, and future. Annu. Rev. Env. Resour. 44, 89-115 (2019).

4. Wee, et al. The integration and application of genomic information in mangrove conservation. Conserv. Biol. 33, 206-209 (2019).

5. Duke, N., Lo, E. \& Sun, M. Global distribution and genetic discontinuities of mangroves-emerging patterns in the evolution of Rhizophora. Trees-Struct. Funct. 16, 65-79 (2002).

6. Ellison, A. M., Farnsworth, E. J. \& Merkt, R. E. Origins of mangrove ecosystems and the mangrove biodiversity anomaly. Global Ecol. Biogeogr. 8, 95-115 (1999).

7. Plaziat, J.-C., Cavagnetto, C., Koeniguer, J.-C. \& Baltzer, F. History and biogeography of the mangrove ecosystem, based on a critical reassessment of the paleontological record. Wetl. Ecol. Manag. 9, 161-180 (2001).

8. Duke, N., Ball, M. \& Ellison, J. Factors influencing biodiversity and distributional gradients in mangroves. Global Ecol. Biogeogr. Lett. 7, 27-47 (1998).

9. Duke, N. Genetic diversity, distributional barriers and rafting continents-more thoughts on the evolution of mangroves. Hydrobiologia 295, 167-181 (1995).

10. Tomlinson, P. B. The botany of mangroves. (Cambridge University press, 1986).

11. Schwarzbach, A. E. \& Ricklefs, R. E. Systematic affinities of Rhizophoraceae and Anisophylleaceae, and intergeneric relationships within Rhizophoraceae, based on chloroplast DNA, nuclear ribosomal DNA, and morphology. Am. J. Bot. 87, 547-564 (2000).

12. Lo, E. Y. Y. Testing hybridization hypotheses and evaluating the evolutionary potential of hybrids in mangrove plant species. $J$. Evol. Biol. 23, 2249-2261 (2010).

13. Takayama, K., Tamura, M., Tateishi, Y., Webb, E. L. \& Kajita, T. Strong genetic structure over the American continents and transoceanic dispersal in the mangrove genus Rhizophora (Rhizophoraceae) revealed by broad-scale nuclear and chloroplast DNA analysis. Am. J. Bot. 100, 1191-1201 (2013).

14. Lo, E., Duke, N. \& Sun, M. Phylogeographic pattern of Rhizophora (Rhizophoraceae) reveals the importance of both vicariance and long-distance oceanic dispersal to modern mangrove distribution. BMC Evol. Biol. 14, 83 (2014).

15. Chen, Y. et al. Applications of multiple nuclear genes to the molecular phylogeny, population genetics and hybrid identification in the mangrove genus Rhizophora. PLoS ONE 10, e0145058 (2015).

16. $\mathrm{Xu}, \mathrm{S}$. H. et al. The origin, diversification and adaptation of a major mangrove clade (Rhizophoreae) revealed by whole-genome sequencing. Natl. Sci. Rev. 4, 721-734 (2017).

17. Tyagi, A. P. Cytogenetics and reproductive biology of mangroves in Rhizophoraceae. Aust. J. Bot. 50, 601-605 (2002).

18. Tyagi, A. P. Chromosomal Pairing and Pollen Viability in Rhizophora mangle and Rhizophora stylosa Hybrids. S. Pac. J. Nat. Sci. 20, 1-3 (2002).

19. Tyagi, A. P. \& Singh, E. V. V. Pollen fertility and intraspecific and interspecific compatibility in mangroves of Fiji. Sex. Plant Reprod. 11, 60-63 (1998). 
20. Steininger, F. F. \& Rögl, F. Paleogeography and palinspastic reconstruction of the Neogene of the Mediterranean and Paratethys. Geol. Soc. Spec. Publ. 17, 659-668 (1984).

21. Harzhauser, M. et al. Biogeographic responses to geodynamics: a key study all around the Oligo-Miocene Tethyan Seaway. Zoo. Anz. 246, 241-256 (2007).

22. Vrielynck, B., Odin, G. \& Dercourt, J. Miocene palaeogeography of the Tethys Ocean; potential global correlations in the Mediterranean. Miocene stratigraphy: an integrated approach. Elsevier Science, (1997).

23. Harzhauser, M., Piller, W. E. \& Steininger, F. F. Circum-Mediterranean Oligo-Miocene biogeographic evolution-the gastropods' point of view. Palaeogeogr. Palaeoclimatol. Palaeoecol. 183, 103-133 (2002).

24. Dercourt, J. et al. Geological evolution of the Tethys belt from the Atlantic to the Pamirs since the LIAS. Tectonophysics 123, 241-315 (1986).

25. Marko, P. B. Fossil calibration of molecular clocks and the divergence times of geminate species pairs separated by the Isthmus of Panama. Mol. Biol. Evol. 19, 2005-2021 (2002).

26. Saenger, P. Mangrove vegetation: an evolutionary perspective. Mar. Freshw. Res. 49, 277-286 (1998).

27. Muller, J. \& Caratini, C. Pollen of Rhizophora (Rhizophoraceae) as a guide fossil. Pollen Spores 19, 361-390 (1977).

28. Muller, J. Fossil pollen records of extant angiosperms. Bot. Rev. 47, 1-142 (1981).

29. Germeraad, J. H., Hopping, C. A. \& Muller, J. Palynology of tertiary sediments from tropical areas. Rev. Palaeobot. Palyno. 6, 189-348 (1968).

30. Zachos, J., Pagani, H., Sloan, L., Thomas, E. \& Billups, K. Trends, rhythms, and aberrations in global climate 65 Ma to present. Science 292, 686-693 (2001).

31. Pole, M. S. \& Macphail, M. K. Eocene Nypa from Regatta Point, Tasmania. Rev. Palaeobot. Palyno. 92, 55-67 (1996).

32. Hornibrook, N. D. B. New Zealand Cenozoic marine paleoclimates: a review based on the distribution of some shallow water and terrestrial biota. Pacific Neogene: environment, evolution, and events, 83-106 University of Tokyo Press, (1992).

33. Hou, Z. \& Li, S. Tethyan changes shaped aquatic diversification. Biol. Rev. 93, 874-896 (2018).

34. Wee, A. K. S. et al. Genetic differentiation and phylogeography of partially sympatric species complex Rhizophora mucronata Lam. and R. stylosa Griff. using SSR markers. BMC Evol. Biol. 15, 57 (2015).

35. Ng, W. L. et al. Closely related and sympatric but not all the same: genetic variation of Indo-West Pacific Rhizophora mangroves across the Malay Peninsula. Conserv. Genet. 16, 137-150 (2015).

36. Doyle, J. \& Doyle, J. A rapid DNA isolation procedure for small quantities of fresh leaf tissue. Phytochem. Bull. 9, 11-15 (1987).

37. Strand, A. E., Leebens-Mack, J. \& Milligan, B. G. Nuclear DNA-based markers for plant evolutionary biology. Mol. Ecol. 6, 113-118 (1997).

38. Cronn, R. C., Small, R. L. \& Wendel, J. F. Duplicated genes evolve independently after polyploid formation in cotton. Proc. Natl. Acad. Sci. USA 96, 14406-14411 (1999).

39. Hayashi, K. PCR-SSCP: a simple and sensitive method for detection of mutations in the genomic DNA. Genome Res. 1, 34-38 (1991).

40. Rozas, J., Sanchez-DelBarrio, J. C., Messeguer, X. \& Rozas, R. DnaSP, DNA polymorphism analyses by the coalescent and other methods. Bioinformatics 19, 2496-2497 (2003).

41. Swofford, D.L. PAUP*. Phylogenetic Analysis Using Parsimony ( ${ }^{\star}$ and Other Methods). Sinauer Associates, Sunderland, Massachusetts, (2002).

42. Stamatakis, A. RAxML version 8: a tool for phylogenetic analysis and post-analysis of large phylogenies. Bioinformatics 30, 13121313 (2014).

43. Leigh, J. W. \& Bryant, D. PopART: Full-feature software for haplotype network construction. Methods Ecol. Evol. 6, 1110-1116 (2015).

44. Bandelt, H. J., Forster, P. \& Röhl, A. Median-joining networks for inferring intraspecific phylogenies. Mol. Biol. Evol. 16, I37-48 (1999).

45. Drummond, A. J. \& Rambaut, A. BEAST: Bayesian evolutionary analysis by sampling trees. BMC Evol. Biol. 7, 214 (2007).

46. Heled, J. \& Drummond, A. J. Bayesian inference of species trees from multilocus data. Mol. Biol. Evol. 27, 570-580 (2009).

47. Graham, A. Paleobotanical evidence and molecular data in reconstructing the historical phytogeography of Rhizophoraceae. Ann. Mo. Bot. Gard. 93, 325-334 (2006).

48. Rambaut, A. Fig Tree v1.4. (2012). Available at http://tree.bio.ed.ac.uk/software/figtree/

49. Matzke, N. J. Probabilistic historical biogeography: new models for founder-event speciation, imperfect detection, and fossils allow improved accuracy and model-testing. Front. Biogeogr. 5, 242-248 (2013).

50. Blair, C. \& He, X. J. RASP 4: ancestral state reconstruction tool for multiple genes and characters. Mol. Biol. Evol. 37, 604-606 (2020).

51. Takayama, K., Tamura, M., Tateishi, Y. \& Kajita, T. Isolation and characterization of microsatellite loci in a mangrove species, Rhizophora stylosa (Rhizophoraceae). Conserv. Genet. Resour. 1, 175-178 (2009).

52. Takayama, K., Tamura, M., Tateishi, Y. \& Kajita, T. Isolation and characterization of microsatellite loci in the red mangrove Rhizophora mangle (Rhizophoraceae) and its related species. Conserv. Genet. 9, 1323-1325 (2008).

53. Pritchard, J. K., Stephens, M. \& Donnelly, P. Inference of population structure using multilocus genotype data. Genetics 155, 945-959 (2000).

54. Falush, D., Stephens, M. \& Pritchard, J. K. Inference of population structure using multilocus genotype data: dominant markers and null alleles. Mol. Ecol. Notes. 7, 574-578 (2007).

55. Hubisz, M. J., Falush, D., Stephens, M. \& Pritchard, J. K. Inferring weak population structure with the assistance of sample group information. Mol. Ecol. Resour. 9, 1322-1332 (2009).

56. Evanno, G., Regnaut, S. \& Goudet, J. Detecting the number of clusters of individuals using the software structure: a simulation study. Mol. Ecol. 14, 2611-2620 (2005).

\section{Acknowledgements}

This work was supported by the Japan Society for the Promotion of Science (JSPS) KAKENHI (JP22405005, JP25290080, and JP17H01414 to TK) and Fujiwara Natural History Foundation and KAKENHI JP07J02524 to KT. Field work was partially supported by JSPS KAKENHI (JP14405015 to YT) and a grant for aid by Yamada Science Foundation 2005 (to TK). We thank Drs Y. Abe, Alessandro, G. Ameka, C. Baider, M. Bovini, H.C. de Lima, G. Flora, J.A. Jiménez, R. Nagahisma, S. Nakata, E. Narvaez, D. Neil, K. Watanabe-Toma, A. Santiágo, H. Takayama, N. Wakita, JBRJ (Brazil), CNPq (Brazil), MPEG (Brazil), MINAE (Costa Rica), OTS (Costa Rica), QCNE (Ecuador), STRI (Panama), MEXU (Mexico) and NOU (New Caledonia) for their support in field sampling. 


\section{Author contributions}

K.T., Y.T., and T.K. have made substantial contributions to the conception and design of the work and field survey. K.T. conducted molecular analyses. K.T. and T.K. discussed the interpretation of data and drafted the manuscript. K.T., Y.T., and T.K. substantively revised it.

\section{Competing interests}

The authors declare no competing interests.

\section{Additional information}

Supplementary Information The online version contains supplementary material available at https:/doi.org/ 10.1038/s41598-021-85844-9.

Correspondence and requests for materials should be addressed to K.T. or T.K.

Reprints and permissions information is available at www.nature.com/reprints.

Publisher's note Springer Nature remains neutral with regard to jurisdictional claims in published maps and institutional affiliations.

Open Access This article is licensed under a Creative Commons Attribution 4.0 International License, which permits use, sharing, adaptation, distribution and reproduction in any medium or format, as long as you give appropriate credit to the original author(s) and the source, provide a link to the Creative Commons licence, and indicate if changes were made. The images or other third party material in this article are included in the article's Creative Commons licence, unless indicated otherwise in a credit line to the material. If material is not included in the article's Creative Commons licence and your intended use is not permitted by statutory regulation or exceeds the permitted use, you will need to obtain permission directly from the copyright holder. To view a copy of this licence, visit http://creativecommons.org/licenses/by/4.0/.

(C) The Author(s) 2021 